\title{
APLICAÇÃO DAS GEOTECNOLOGIAS NA PRODUÇÃO DE EXERCÍCIOS VOLTADOS PARA O ENSINO DE GEOGRAFIA: UM ESTUDO DE CASO PARA A ILHA DO GOVERNADOR - RJ
}

\section{Application of Geotechnologies in the Production of Exercises to Geography Teaching: a case study for a Ilha do Governador - RJ}

\author{
Barbara Figueiredo de Oliveira \\ Graduanda no Departamento de Geografia da FFP UERJ \\ barbarageosig@yahoo.com.br
}

Fabiana Lopes Ferreira Mestranda no Programa de Pós Graduação em Geografia da FFP UERJ fabianalopesferreira@yahoo.com.br

\author{
Vinicius da Silva Seabra \\ Professor Adjunto do Departamento de Geografia da FFP UERJ \\ vinigeobr@yahoo.com.br
}

Artigo recebido em 26/10/2015 e aceito para publicação em 17/12/2015

DOI: $10.12957 /$ tamoios.2015.20806

Resumo

Abstract
Este trabalho tem como objetivo a utilização do Google Earth para a produção de materiais didáticos voltados para o ensino de Geografia, sobretudo de temas de Geomorfologia e espaço geográfico, para estudantes do ensino fundamental 2, que vivem na Ilha do Governador, município do Rio de Janeiro. O uso de imagens extraídas do Google Earth, em diferentes escalas, permite aos alunos uma interpretação mais completa de suas vizinhanças, além de possibilitar a integração espacial de diferentes componentes espaciais com a Baia de Guanabara. É interessante notar que a utilização desta ferramenta facilita a visualização oblíqua e vertical do espaço, e ainda em perspectiva $2 \mathrm{D}$ e $3 \mathrm{D}$, bem como a abordagem de temas geográficos de uma forma dinâmica e interdisciplinar. A escolha dos temas foi baseada no estudo dos Parâmetros Curriculares Nacionais (PCN), e também sobre os conteúdos considerados importantes para os alunos das escolas de Ilha do Governador. As atividades foram construídas em folhas A4 a um baixo custo, e aplicadas para introduzir os temas a serem discutidos nas aulas de geografia, tornando possível ainda a revisão do conhecimento prévio dos alunos, além de contribuir no desenvolvimento de habilidades voltadas para análise de dados espaciais.

Palavras chave: Google Earth, Sensoriamento Remoto, Exercícios Práticos

This paper aims to use Google Earth for the production of teaching materials devoted to the teaching of geography, mainly geomorphology of subjects and geographic space for elementary school students, living on Ilha do Governador, in Rio de Janeiro municipality. The use of images taken from Google Earth, at different scales, allows students a more complete interpretation of their neighborhoods, and enable spatial integration of different spatial components with the Guanabara Bay. Interestingly, the use of this tool facilitates the oblique and vertical viewing space, plus 2D and 3D perspective as well as the approach of geographic issues in a dynamic and interdisciplinary way. The choice of topics considered the study of the National Curriculum Parameters (PCN), and about the knowledge considered important for students from Governor's Island schools. The activities were built on A4 sheets at a low cost, and applied to introduce the topics to be discussed in geography classes, making it possible still reviewing the students' prior knowledge, and contribute to the development of focused skills for spatial data analysis.

Keywords: Google Earth, Remote Sensing, practical drills. 


\title{
INTRODUÇÃO
}

Vivenciamos um acelerado desenvolvimento tecnológico voltado para novas formas de comunicação e maior utilização de ferramentas de representação, consulta e análise espacial. Nos meios científicos e acadêmicos observamos a disseminação destas inovações, chamadas de geotecnologias, que permitem a aquisição, processamento e manipulação de dados espaciais, além do armazenamento e apresentação dessas informações em forma de gráficos, mapas, tabelas etc. Dentro deste contexto, torna-se imprescindível a maior utilização destas ferramentas no ensino básico de Geografia, seja na produção de materiais didáticos, nas atividades práticas ou como meio de compreensão de um problema espacial.

Nesta perspectiva, Carvalho (2012) ressalta o potencial do sensoriamento remoto para essa ciência, ao apresentar uma gama de vantagens e apontar grandes avanços para a observação, análise e compreensão do espaço vivido, nas suas variadas escalas. Para essa autora, além das fotografias aéreas e das imagens de satélite exibirem um grau de abstração bem menor que os mapas tradicionais, o uso de áreas familiares aos alunos favorece o reconhecimento e observações mais claras, o que beneficia trabalhos posteriores de representação e análise desse espaçogeográfico.

Outra vantagem é a atualização constante das informações, permitindo o estudo das transformações do espaço geográfico ao se observar as mudanças ocorridas através de comparações entre imagens mais recentes e mais antigas. Assim, ela assegura que:

\begin{abstract}
A partir das comparações o aluno pode "ver" esse espaço geográfico atualizado, observando-o, comparando e identificando suas transformações, o que causa grande impressão nos alunos e, a partir dos dados coletados por meio destas observações, pode se criar a necessidade de se questionar, analisar e refletir sobre como a realidade se apresenta (CARVALHO, 2012, p.34).
\end{abstract}

Dessa forma, percebemos que o uso das geotecnologias para a produção de material didático para o ensino de Geografia tem grande importância no trabalho com as questões espaciais em escala local e regional. Os livros didáticos utilizados pelos professores em sala de aula apresentam conteúdos que se distanciam muito da realidade dos alunos, recebendo a questão local pouca atenção nesses materiais. Assim, o acesso e uso dos produtos gerados pelas geotecnologias tornam-se essenciais tendo em vista que facilitam abordagens mais significativas para os alunos e favorecem o processo de ensino-aprendizagem em Geografia (CONCEIÇÃO et al, 2009).

Entre os softwares mais conhecidos, Google Earth apresenta-se como uma 
ferramenta de grande valia para essa disciplina. Além de ser gratuito e estar presente nos diversos meios de comunicação, permite a visualização da superfície terrestre em diferentes escalas, em modelos tridimensionais do terreno e em uma perspectiva multitemporal (a partir de imagens de diferentes anos) (MARTINS et al, 2013). Esta ferramenta permite a construção de representações em escala local, trabalhando o espaço vivido dos alunos, auxiliando na compreensão de diferentes temas em Geografia. Destaca-se que estes recursos possibilitam a visualização do espaço em visão obliqua e vertical, em perspectiva 2D e 3D e em diferentes escalas, bem como a abordagem de temas geográficos de forma dinâmica einterdisciplinar.

Portanto, este trabalho tem como objetivo apresentar a potencialidade do uso de imagens de Sensoriamento Remoto em sala de aula, fundamentando-se no uso do Google Earth como ferramenta para o desenvolvimento de materiais didáticos em Geografia, voltados para alunos do $6^{\circ}$ do Ensino Fundamental II da Ilha do Governador. Almeja-se o desenvolvimento de atividades práticas que possibilitem abordagens multidisciplinares e que tornem mais palpáveis para os alunos o tema abordado, muitas vezes considerado abstrato.

\section{METODOLOGIA}

A escolha dos temas baseou-se no estudo dos Parâmetros Curriculares Nacionais (PCN), onde especifica que a geografia deve ser um meio de leitura e compreensão do mundo, bem como, a abordagem da modernização, do modo de vida e da problemática ambiental, constados no quarto ciclo, do Eixo 3, e em conteúdos importantes para os alunos de escolas da Ilha do Governador, localizada no município do Rio de Janeiro. As atividades foram construídas em pranchas de folha A4, a baixo custo, para serem aplicadas na introdução dos conteúdos geográficos, pois o uso das imagens de satélite permite verificarmos os conhecimentos prévios dos alunos, preparando para que eles estabeleçam novas relações e tenham condições de captar dados e elaborar respostas. Ao final da elaboração das pranchas de exercícios será confeccionado um guia de atividades práticas com o uso do Google Earth. As etapas de desenvolvimento do trabalho podem ser enunciadas abaixo:

1. Pesquisa bibliográfica e seleção de conteúdos geográficos a partir dos Parâmetros Curriculares Nacionais (PCN), compatíveis com as atividades com potencialidade de realização o Google Earth. 
2. Exportação de dados vetoriais de plataformas SIG para o formato KML, e sua posterior importação para o Google Earth.

3. Elaboração de pranchas de exercícios dentro de uma proposta multidisciplinar, com enfoque de abordagem em Geografia, para posterior aplicação em sala deaula.

4. Elaboração final do Guia de Atividades Práticas com o Uso do GoogleEarth.

$\mathrm{Na}$ elaboração de todas as etapas do trabalho, é importante destacar a importância de duas ferramentas, que são o Google Earth e o Google Maps. Segundo Carvalho et al. (2014), o Google Earth e o Google Maps são ferramentas que permitem a visualização de qualquer local da Terra a partir de imagens de satélite, mapas e modelos tridimensionais do terreno. Além de permitirem acesso gratuito, estas ferramentas possibilitam o trabalho de temas diversos, em diferentes escalas e ainda numa perspectiva multitemporal (com imagens de diferentes datas).

Além da visualização de imagens e modelos do terreno em diferentes escalas, podemos acessar fotografias, bases digitais, e dados de enciclopédias. Pode-se ainda trabalhar com sistemas de coordenadas em diferentes unidades de referência, o movimento aparente do sol (fusos horários), imagens orbitais do passado, percepção do terreno em diferentes pontos de vista (visão de topo e oblíqua), dados de superfície e do leito oceânico, imagens de satélites geoestacionários, dentre outras ferramentas (CARVALHO et al., 2014).

\section{RESULTADOS}

Ao todo foram desenvolvidas quatro pranchas de exercícios, em tamanho A4, as quais serão aplicadas, no decorrer do ano, em turmas de ensino fundamental II na Ilha do Governador, município do Rio de Janeiro. Todas serão impressas em papel glosser ou fotográfico e poderão ser reutilizadas em cada atividade. Em geral, as pranchas têm a pretensão de trabalhar os temas de forma multiescalar com a finalidade de abranger o espaço vivido dos alunos, contextualizando a Ilha do Governador em relação à Baia de Guanabara. 
Pesquisa Bibliográfica e

seleçāo de conteúdos

geográficos, a partir do

PCN, compativeis com

atividades disponiveis no

Google Earth
Utilizaçāo de dados

exportados do ArcGis em

KML

Elaboraçăo de Pranchas de

Exercicios em Geografia

dentro de uma proposta

Multidisciplinar, que possa

ser aplicada em sala de

aula

Confecçāo final do Guia de

Atividades Práticas e:

Multidisciplinares com o

uso do Google Earth

Figura 1. Metodologia utilizada para a produção do materialdidático

Neste contexto, apresentamos três das atividades desenvolvidas nesta proposta com as figuras, a partir de sua problematização, seus objetivos e resultados esperados. A primeira e a segunda atividade (figuras 2,3 e 4) tiveram como principal objetivo discutir os conceitos de relevo, baía e bacias hidrográficas, ressaltando ainda o funcionamento sistêmico e interdependente de ambos, no contexto da Baía da Guanabara, discutindo ainda seu atual estado de conservação. A terceira atividade tem como objetivo discutir e pontuar as mudanças na urbanização em Cocotá, bairro da Ilha do Governador, nos últimos anos e a influência dos meios de transporte nessas mudanças.

Os resultados esperados com as pranchas 1 e 2 giram entorno da expectativa de discutir os conceitos propostos dentro de uma perspectiva sistêmica, chamando atenção ainda para o fato de que o atual estado de conservação da Baía da Guanabara é reflexo da poluição dos rios que nela deságuam. É importante ainda que o aluno chegue à conclusão que hoje as praias da Ilha do Governador são poluídas justamente por estarem inseridas no interior da Baía da Guanabara, e não somente pela poluição gerada pelas atividades econômicas presentes na própria Ilha. 


\section{ATIVIDADE 1}

Você sabia que a Baia de Guanabara possui aproximadamente $380 \mathrm{~km}^{2}$ de extensão, sendo a segunda maior Baia do Brasil, e que em seu interior deságuam 55 rios e riachos?

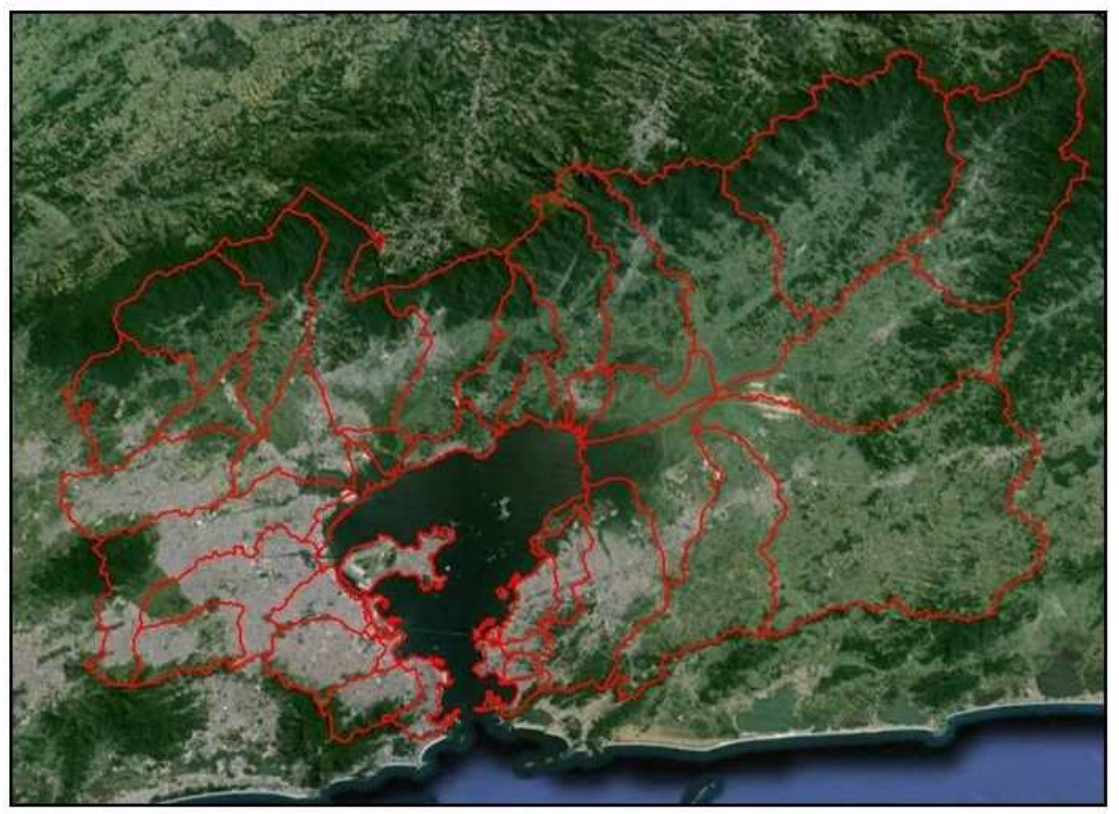

Você sabia que esses rios e riachos passam pela segunda maior metrópole do Brasil, levando em suas águas muito lixo e esgoto?

Agora que você já conhece um pouco da nossa área de estudo, pesquise um pouco mais e escreva um pequeno texto, de até 15 linhas, explicando o que você acha que deve ser feito para despoluirmos a Baía de Guanabara!

Figura 2. Prancha da delimitação da Bacia da Baia de Guanabara

\section{Atividade 2}

O relevo do entorno da baía de Guanabara é muito diverso. Podemos encontrar nos seus limites, ao norte, a escarpa da Serra do Mar e também suas montanhas. Também temos os maciços da Tijuca, Pedra Branca, Gericinó-Mendanha, e etc.

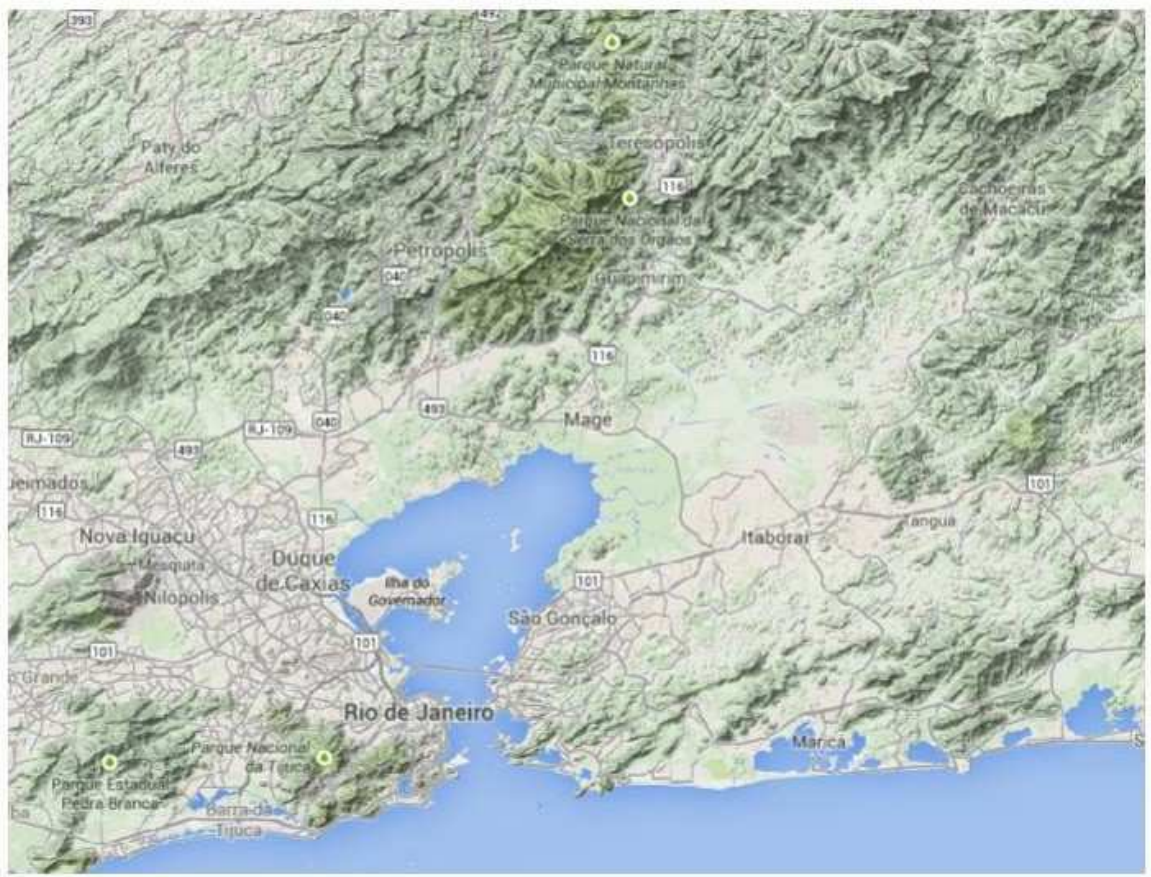

Temos também as grandes planícies e morros, que são as áreas mais ocupadas da região metropolitana.

Sabendo que as áreas mais escuras são áreas onde o relevo é mais acentuado, construa uma maquete de massinha do relevo do da bacia da Baía de Guanabara e seu entorno. Se necessário utilize a prancha auxiliar para visualizar melhor o relevo e a delimitação da bacia da Baia de Guanabara.

Figura 3. Prancha para elaboração da maquete 
As pranchas $3 \mathrm{a}$ e $3 \mathrm{~b}$ representadas pelas figuras 4 e 5 almejam obter a compreensão dos alunos sobre temas voltados para a urbanização, a organização espacial do bairro, a partir da comparação das mudanças no espaço urbano em um período de 10 anos, além de trabalhar a interação das mudanças desse espaço com as redes, tendo como principal exemplo nessa localidade o terminal da Barcas S.A. no bairro do Cocotá, na Ilha do Governador. No enunciado da questão, tomamos a problematização da seguinte maneira: Nos últimos dez anos grandes obras beneficiaram o bairro de Cocotá, localizado na Ilha do Governador. Compare as duas imagens (2003 e 2013) e identifique as possíveis transformações ocorridas no espaço nesse período.

A utilização dessas pranchas como material de apoio facilita aos alunos à leitura dos principais alvos das imagens, que são: as áreas de cobertura vegetal (verde), as áreas urbanas (cinza), as áreas de gramíneas (verde claro) e os corpos hídricos (preto ou azul). Pode-se mostrar que a bacia em seus principais divisores nas áreas de montanhas, e que todas as bacias (delimitadas em vermelho) deságuam na Baía de Guanabara. Essa prancha apresenta ainda a potencialidade de discutir o conceito de metrópole e de situar a Ilha do Governador neste contexto, sendo importante ressaltar que na bacia da Baía de Guanabara existem muitas indústrias, que grande parte do esgotamento sanitário não é tratado.

Nas pranchas utilizadas na atividade 2 (figuras 3 e 4) ressalta-se a importância de explicar que as áreas de sombreamento (textura) são as áreas de relevo movimentado (morros ou montanhas), onde a prancha auxiliar facilita explicação desse conteúdo além de subsidiar a construção da maquete do relevo da bacia da Baia de Guanabara e seu entorno.Essa atividade ainda possibilita trabalhar a visão $2 \mathrm{D}$ e $3 \mathrm{D}$, obliqua e vertical, tornando mais palpável para os alunos conceitos que muitas vezes são complexos por serem abstratos.

É importante ressaltar que todas as atividades, como sugere Carvalho (2012), deverão ser aplicadas na introdução dos temas a serem discutidos, pois o uso das imagens de satélite cria a oportunidade de verificarmos os conhecimentos prévios dos alunos, preparando o campo para que eles venham a estabelecer novas relações e tenham mais condições de captar dados e elaborar respostas. 
Prancha auxiliar: atividade 2
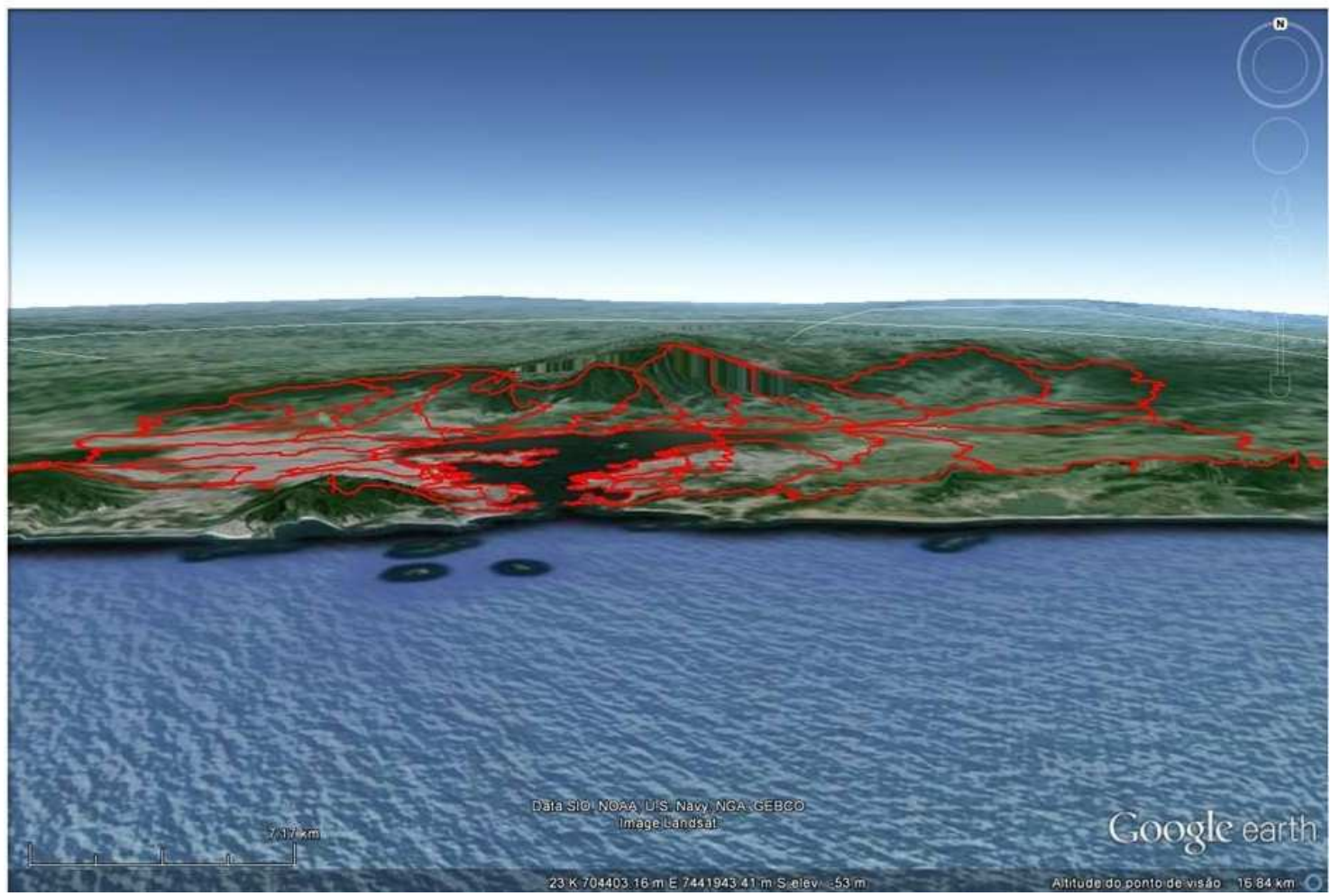

Figura 4. Prancha para auxiliar na atividade prática 2.

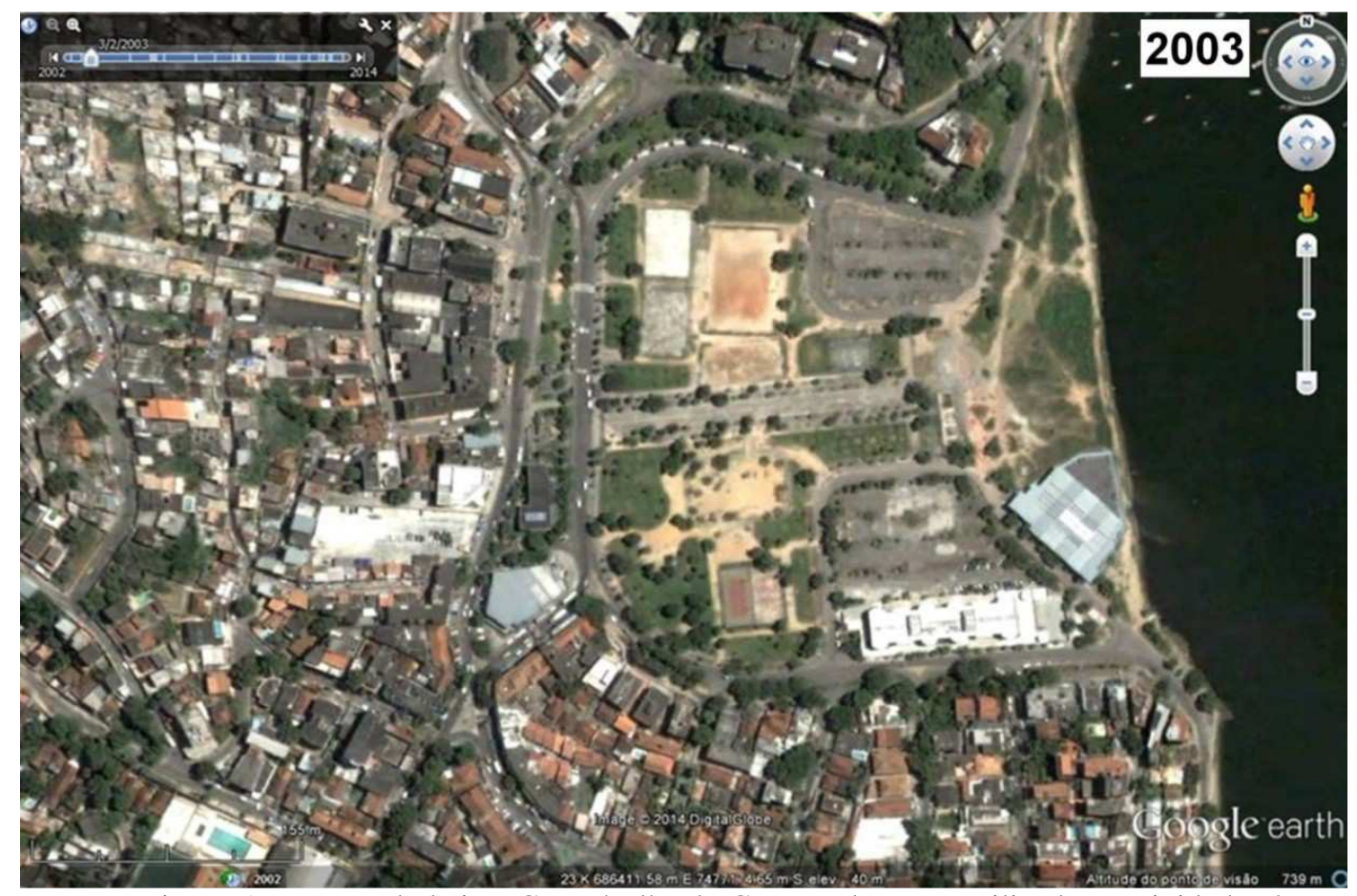

Figura 5. Imagem do bairro Cocotá, Ilha do Governador - RJ, utilizada na atividade três. Imagem extraída do Google Earth, 03/02/2003. 


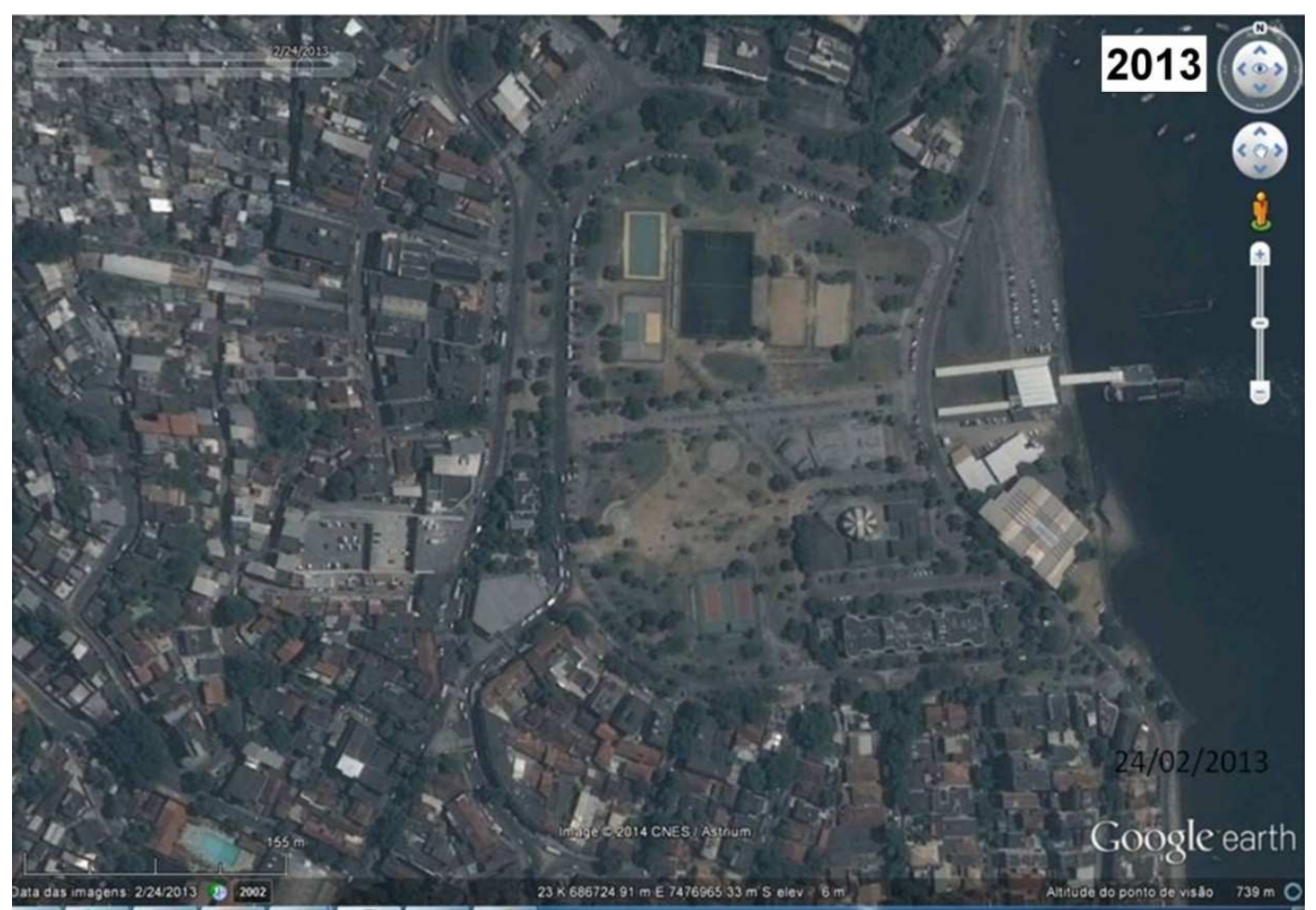

Figura 6. Imagem do bairro Cocotá, Ilha do Governador - RJ, utilizada na atividade três. Imagem extraída do Google Earth, 23/02/2013.

Além disso, os trabalhos propostos procuram preparar os alunos para a aprendizagem dos conceitos elementares da cartografia, partindo da construção de um mapa pelos alunos, durante a qual foram sendo discutidas todas as necessidades envolvidas na tarefa, como por exemplo, a necessidade da legenda, a noção de escala, os pontos de orientação, entre outros conceitos.

\section{CONCLUSÕES}

É relevante destacar a importância de se trabalhar com imagens da área do entorno da escola, ou seja, a área de vivência dos alunos. Esperamos que em todas as atividades propostas o conhecimento prévio destes alunos em relação às imagens seja um grande facilitador e motivador da aprendizagem, tornando-a mais rápida e efetiva. Além disso, esperamos que as atividades permitam uma maior interação, colocando os alunos em uma posição ativa, de colaboradores nas tarefas o que pode contribuir para torná-las mais significativas e permita a construção dos conceitos trabalhados, diferentemente do ocorre quando se utilizam as práticas correntes que remetem à mera memorização.

Os exercícios propostos neste trabalho facilitam a compreensão dos alunos ao perceber a localização da Ilha do Governador no contexto da metrópole do Rio de 
Janeiro, assim como entender alguns conceitos geomorfológicos dessa área e que permitem a análise das interferências, não só dos insulanos, como de todos os municípios ao entorno na Baia de Guanabara. Por se tratar de uma ilha, inserida dentro de uma das maiores baías do Brasil, a discussão de conceitos geomorfológicos a partir das imagens de satélite se faz necessária a todo o momento, sendo ainda uma importante oportunidade de reduzir as abordagens fragmentadas da geografia, já que podemos trabalhar de forma articulada a geomorfologia com temas sociais e urbanos. É relevante ainda mencionarmos que as representações gráficas são importantes ferramentas para Geografia, já que descrevem, em diferentes escalas e níveis de generalização, aspectos importantes do espaço que devem ser compreendidos e analisados, servindo em muitas situações como o ponto de partida para uma investigação geográfica. Ou seja, para explicarmos a razão da localização da ocorrência de fenômenos ou objetos na superfície terrestre, devemos antes de tudo visualizar (ler) a disposição ou organização destes elementos no espaço. Desta maneira, torna-se relevante a realização de práticas e outras estratégias que contribuam para a construção leitura das representações gráficas do espaço geográfico.

\section{REFERÊNCIAS BIBLIOGRÁFICAS}

BRASIL. Parâmetros curriculares nacionais. Geografia. Brasília: MEC/SEF, 1998.

CARVALHO, V. S. G. Sensoriamento remoto no ensino básico da Geografia: definindo estratégias. Rio de Janeiro: APED, 2012.

CARVALHO, V. S. G; RICHTER, M. CRUZ, C. B. M.; SEABRA, V. S. O Uso de Geotecnologias livres no ensino básico: Uma proposta de implementação e avaliação. In: De baixo para cima: política e tecnologia na educação. Org: Tamara Tania Cohen Egler. RPPE. Rio de Janeiro. Letra Capital. pag. 161-176. 2014

CONCEIÇÃO, T. S.; FILHO, S. F. C.; CUNHA, A. S. O sensoriamento remoto no ensino de geografia: A produção e aquisição de material didático e sua aplicação em sala de aula. In: Simpósio Brasileiro de Sensoriamento Remoto (SBSR), 14., 2009, Natal. Anais... São José dos Campos: INPE, 2005. Artigos, p. . CD-ROM, On-line. ISBN 85-17-00018-8. Disponível em: http://sbsr.dpi.inpe.br/col/dpi.inpe.br/sbsr\%4080/2008/11.18.01.21.37/doc/sbsr2009.pdf. Acesso em: 30 set. 2014.

MARTINS, L. J.; SEABRA, V. S.; CARVALHO, V. S. G. O uso do Google Earth como ferramenta no ensino básico da Geografia. In: Simpósio Brasileiro de Sensoriamento Remoto (SBSR), 16., 2013, Foz do Iguaçu. São José dos Campos: INPE, 2013. Artigos, p. 2657-2664. CD-ROM, On-line. ISBN 85-17-00018-8. Disponível em: http://www.dsr.inpe.br/sbsr2013/files/p0251.pdf. Acesso em: 30 set. 2014.

RICHTER, M; SOUSA, G. M.; SEABRA, V. S. O Desafio do Ensino de Geotecnologias. In: Aprendendo Geografia: Reflexões Teóricas e Experiências de Ensino da UFRRJ. Cristiane Cardoso e Leandro Dias de Oliveira (Orgs.). 2012. 